\title{
DESCRIÇÃO MORFOLÓGICA DE FRUTOS E SEMENTES DE Manilkara salzmannii (SAPOTACEAE)
}

\author{
Eduardo Bezerra de Almeida Jr. ${ }^{1}$, Liliane Ferreira Lima ${ }^{2}$, Patrícia Barbosa Lima ${ }^{2}$, Carmen Sílvia Zickel ${ }^{3}$ \\ ${ }^{1}$ Biólogo, M.Sc., Doutorando em Botânica, UFRPE, Recife, PE, Brasil - ebaj25@ yahoo.com.br \\ ${ }^{2}$ Bióloga, Mestranda no Pós-Graduação em Botânica, UFRPE, Recife, PE, Brasil - lilianef.lima@ gmail.com, \\ patriciablima@gmail.com \\ ${ }^{3}$ Bióloga, Dra ., Depto. de Biologia, UFRPE, Recife, PE, Brasil - zickelbr@yahoo.com \\ Recebido para publicação: 17/02/2009 - Aceito para publicação: 09/02/2010
}

\begin{abstract}
Resumo
O gênero Manilkara possui importância econômica considerável, devido aos frutos comestíveis, qualidade da madeira e látex, podendo ser usada na medicina popular e ornamentação. O gênero está representado no Brasil por aproximadamente 19 espécies, distribuídas com maior representatividade na floresta atlântica e restinga. $\mathrm{O}$ presente estudo teve como objetivo caracterizar a biometria dos frutos e sementes de Manilkara salzmannii (A. DC.) H. J. Lam visando contribuir com dados sobre a biologia e comportamento dessa espécie, fornecendo subsídios importantes para a sua identificação. Os frutos maduros foram coletados manualmente ou com tesoura de poda alta, armazenados em sacos de papelmadeira, identificados e resfriados até o processamento. Para a análise morfológica foram selecionados aleatoriamente 100 frutos e 100 sementes. As sementes foram extraídas dos frutos manualmente, lavadas em água corrente, secas com flanela, e posteriormente pesadas, determinando-se o número de sementes por quilograma e o peso de 1000 sementes. Os frutos e as sementes foram analisados com o intuito de contribuir como uma ferramenta para a diferenciação das espécies, bem como auxiliar na determinação da variabilidade da espécie em estudos de dispersão e dos agentes dispersores, sendo importante também para as análises de sucessão e regeneração de ecossistemas florestais.
\end{abstract}

Palavras-chave: Diásporos; frutos; Manilkara; sementes.

\begin{abstract}
Morphological description of Manilkara salzmannii (Sapotaceae) fruits and seeds. Manilkara gender has a considerable economic value, because of the edible fruits, the quality of its wood, the latex and the fact that it can be used for popular medicine and ornamentation. In Brazil, the gender is represented by approximately 19 species that are mainly distributed through the Atlantic Forest and coastal vegetation. The present study aimed to characterize the biometric aspects of Manilkara salzmannii seeds and fruits in order to contribute to the biology and behavior of the species, and to give important subsidies for its identification. The mature fruits were collected manually or with the help of a pruning shears, stored in paper bags, identified and cooled until processing. A total of 100 fruits and 100 seeds were randomly selected for the morphological analysis. The seeds were manually extracted from the fruits, washed in running water and weighted, in order to determine the number of seeds per kilogram and the weight of a thousand seeds. The fruits and the seeds were analyzed with the aim of contributing as a tool for the differentiation of the species and helping in the determination of the species variability in dispersion studies and dispersion agents. It is also important for the analysis of succession and regeneration of forest ecosystems.
\end{abstract}

Keywords: Diasporas; fruits; Manilkara; seeds.

\section{INTRODUÇÃO}

O gênero Manilkara compreende o quarto maior gênero da família Sapotaceae e está representado no Brasil, por aproximadamente 19 espécies distribuídas em diferentes tipos vegetacionais, com maior representatividade em áreas de Floresta Atlântica e restinga (ANDRADE-LIMA, 1957). Apesar da revisão para a família Sapotaceae, realizada por Pennington (1990), ainda é possível encontrar problemas taxonômicos que são importantes para a delimitação de algumas espécies, sendo necessária a 
realização de estudos que possam contribuir para esclarecer e ampliar o conhecimento desse grupo de plantas, como reporta o autor da revisão.

A espécie Manilkara salzmannii (A. DC.) H. J. Lam, conhecida como maçaranduba, caracterizase por apresentar porte arbóreo-arbustivo, geralmente de 10 a $25 \mathrm{~m}$ de altura, latescente, copa arredondada, tronco ereto e cilíndrico, casca grossa e fissurada longitudinalmente, 40 a $70 \mathrm{~cm}$ de diâmetro. Trata-se de uma planta perenifólia, heliófita e seletiva higrófita (ANDRADE-LIMA, 1957; LORENZI, 1992; PENNINGTON, 1990).

O grupo possui importância econômica, tendo em vista que apresenta madeira de qualidade, podendo ser utilizada na construção civil, na forma de vigas, caibros, tábuas e tacos para assoalhos. O látex pode ser utilizado na produção de gomas, e algumas espécies produzem frutos comestíveis, que podem ser consumidos ou comercializados. Essa espécie pode ainda ser utilizada na medicina popular e também como planta ornamental (RICARDI, 1992). Outra característica importante é o seu uso na arborização e reflorestamento em áreas destinadas à preservação (LORENZI, 1992).

A obtenção de informações sobre as características dos frutos e das sementes de Manilkara salzmannii pode fornecer dados importantes para a identificação e conhecimento do comportamento da espécie nas diferentes regiões ecológicas. Tendo em vista os problemas taxonômicos apresentados pelo grupo e a escassez de estudos específicos focados na flora brasileira, a caracterização biométrica de frutos e sementes constitui uma ferramenta indispensável para a diferenciação de espécies de um mesmo gênero (CRUZ et al., 2001). Além disso, a identificação de frutos e sementes do interior da floresta serve de base para estudos direcionados à germinação, teste de qualidade e armazenamento, entre outros (AMORIN et al., 1997).

Diante do exposto, o presente estudo teve como objetivo a caracterização da biometria do fruto e da semente de Manilkara salzmannii, visando à obtenção de dados complementares voltados para o conhecimento da biologia dessa espécie.

\section{MATERIAL E MÉTODOS}

\section{Local de estudo e coleta do material}

O material coletado foi obtido em fevereiro de 2008, diretamente da copa de cinco árvores matrizes existentes em uma área com floresta de restinga, na Reserva Particular do Patrimônio Natural (RPPN) Nossa Senhora do Outeiro de Maracaípe, localizada no município de Ipojuca, PE (08 $31^{\circ}$ ' $48^{\prime}$ 'S e $35^{\circ} 01^{\prime} 48^{\prime}$ 'W). O clima local é do tipo As, segundo o sistema de classificação de Köppen (1948), com precipitação anual de $2000 \mathrm{~mm}$, com período chuvoso ocorrendo entre maio e julho e período seco entre outubro e dezembro (MEDEIROS et al., 2007).

\section{Coleta e análise do material}

Aproximadamente 1.000 frutos de Manilkara salzmannii foram coletados manualmente ou com o auxílio de tesouras de poda alta, em cinco árvores matrizes. Após a coleta, os frutos foram acondicionados em sacos de polietileno e transportados ao Laboratório de Florística de Ecossistemas Costeiros (LAFLEC), da Universidade Federal Rural de Pernambuco (UFRPE), onde foram transferidos para sacos de papel-madeira, sendo, antes do seu processamento, identificados e resfriados.

Para a análise morfológica (externa e interna) e medição, foram selecionados aleatoriamente 100 frutos e 100 sementes sadios, inteiros, sem deformação, maduros ou em estágio final de maturação. A descrição foi baseada em Barroso et al. (1999).

As sementes utilizadas foram extraídas dos frutos de forma manual, lavadas em água corrente, visando eliminar o látex existente, e secas com uma flanela. Posteriormente, elas foram pesadas em balança digital de precisão (BEL ENGINEERING - Umark 210A), sendo determinado o número de sementes por quilograma e o peso de 1000 sementes, utilizando-se 10 subamostras de 100 sementes cada, segundo as Regras para Análise de Sementes (BRASIL, 1992).

Adotou-se para os frutos e para as sementes o comprimento como a distância entre a base e o ápice, a largura como o maior lado e a espessura como o lado mais estreito. Para auxiliar as medições, utilizou-se um paquímetro digital (Digimess-100.174BL), sendo as observações realizadas com lupa de mesa estereoscópica (Leica Zoom-2000). 


\section{RESULTADOS E DISCUSSÃO}

\section{Morfologia do fruto}

Conforme resultados obtidos, Manilkara salzmannii possui fruto do tipo bacoide (baga), indeiscente, com 14,5-23,6 $\mathrm{mm}$ de comprimento e 16,1-28,6 $\mathrm{mm}$ de largura, de monospermos a oligospermos, com uma a quatro sementes. $\mathrm{O}$ fruto possui odor característico, devido à presença de saponinas triterpenoides, sabor adocicado e agradável quando maduro e adstringente quando imaturo, tendo em vista a grande quantidade de látex de coloração esbranquiçada. Consistência carnosa, polpa bem desenvolvida e abundante, de origem placentar, a qual preenche toda a cavidade interna, onde, na parte central, estão dispostos os lóculos, nos quais se encontram as sementes. Apresenta forma globosa, com epicarpo de textura firme, pouco espessado, liso, glabro, brilhante, de coloração esverdeada quando imaturo, amarelo-alaranjado na fase de maturação e vermelho-alaranjado quando maduro (Figura 1A e 1B). A testa é de consistência dura, quebrando-se quando forçada. Em sua parte interna há uma rede de cordões fibrosos esbranquiçados. O cálice é persistente no fruto, com seis sépalas, às vezes caducas, dispostas em duas séries alternas, com três sépalas externas e três no plano mais interno. O pedicelo apresenta indumento ferrugíneo, às vezes glabrescente (Figura 1C e 1D).

Araújo et al. (2008), no estudo das características físicas e químicas do fruto dessa espécie, afirmaram que o número de sementes por fruto variou de um a quatro, com $37 \%$ dos frutos apresentando uma semente, $9 \%$ apresentando duas e $2 \%$ dos frutos apresentando três ou quatro sementes, comprovando o número de sementes encontradas nos frutos analisados. De acordo com os autores, a espécie possui dificuldade de disseminação, por seus frutos conterem em média uma semente. Ainda segundo eles, o tamanho do fruto não apresenta influência na quantidade de sementes existentes em seu interior. A baixa taxa de germinação de sementes para o gênero Manilkara também foi observada em um estudo sobre germinação de espécies lenhosas em uma área de restinga em Pernambuco (L. F. Lima, dados não publicados), devido à baixa produção de sementes por fruto e à presença de látex, que, segundo a autora, podem dificultar tanto a propagação quanto a obtenção de mudas. Além disso, não houve relação entre variação do tamanho dos frutos e o número de sementes por fruto.

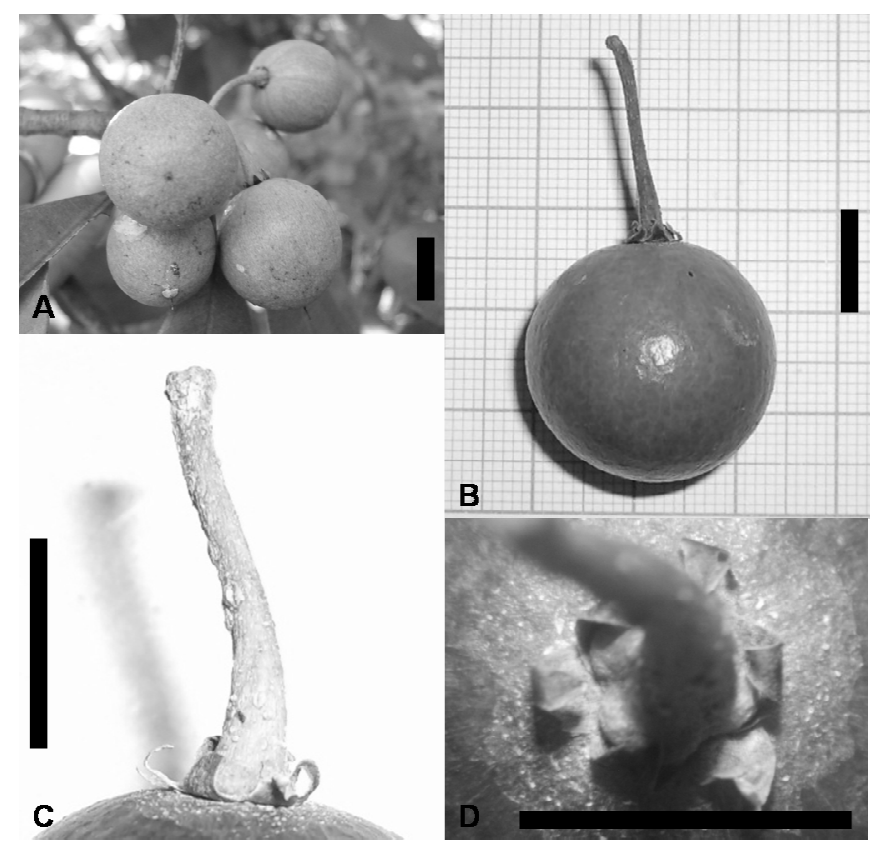

Figura 1. Frutos de Manilkara salzmannii (A. DC.) H. J. Lam: 1A e 1B - frutos imaturos e maduros, respectivamente. $1 \mathrm{C}$ e $1 \mathrm{D}$ - detalhe do pedicelo e do cálice. Barra: $1 \mathrm{~cm}$.

Figure 1. Fruits of Manilkara salzmannii (A. DC.) H. J. Lam: 1A and 1B - immature and mature fruits, respectively. $1 \mathrm{C}$ and $1 \mathrm{D}-$ pedicel and sepals detail. Scale: $1 \mathrm{~cm}$. 


\section{Morfologia da semente}

As sementes apresentam forma elipsoide e estão envolvidas por polpa sucosa. A testa é brilhante, lisa ou com leves ondulações, glabra, bicolor, com coloração que varia de marrom-escuro a castanhoclaro, e que, apesar de dura, quebra-se quando forçada. Em sua parte interna há uma rede de cordões fibrosos. Apresentam dimensões de 10,6-15,4 mm de comprimento, 7,4-9,7 mm de largura e 4-5,4 mm de espessura (Figura 2A e 2B). O hilo é saliente, ventral-basal, obovoide, bem evidente e perceptível a olho nu. Micrópila pequena, subglobosa, geralmente com coloração mais clara que o tegumento. $\mathrm{Na}$ extremidade oposta do hilo, observa-se uma saliência, mais escura que a testa, que pode ser interpretada como a calaza da semente.

Internamente a semente possui um embrião cotiledonar, reto, axial, plano, esbranquiçado (Figura 2C) e presença de endosperma esbranquiçado. A delimitação entre o eixo hipocótilo radicular e os cotilédones é bem visível. Os cotilédones são grandes (8-9,7 mm de comprimento), carnosos, lisos, arredondados, justapostos, plano-convexos (Figura 2D). Eixo hipocótilo-radicular, branco, reto e longo $(2,9-3,8 \mathrm{~mm})$.

Em relação ao peso das sementes, os valores variaram de $0,21-0,46 \mathrm{~g} \pm 0,03$. Os dados obtidos revelaram que as sementes não apresentaram variação acentuada em relação ao peso. Quanto ao peso de 1000 sementes, o valor foi de $271,04 \mathrm{~g}$, o que permite inferir que um quilograma de sementes de Manilkara salzmannii contém, aproximadamente, 3.689 sementes.

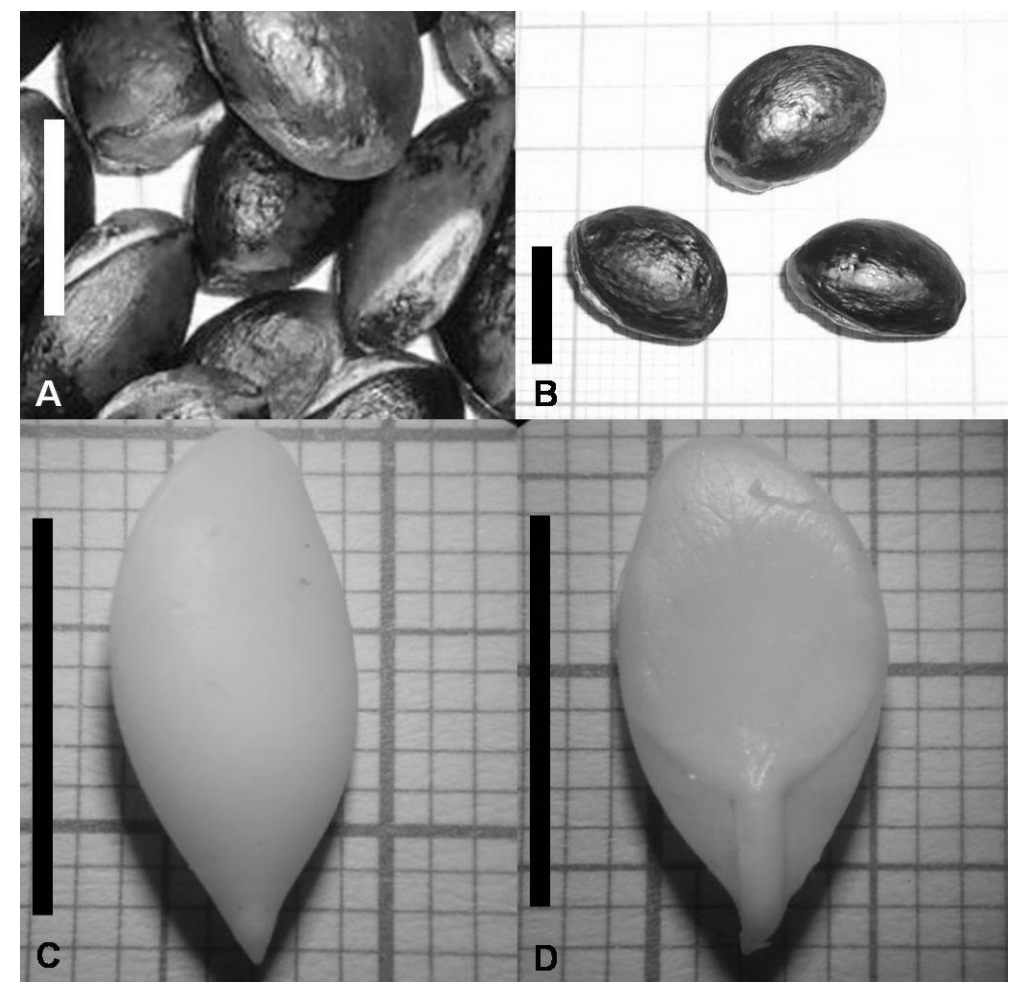

Figura 2. Sementes de Manilkara salzmannii (A. DC.) H. J. Lam: 2A e 2B - detalhe da semente; 2C embrião; 2D - cotilédones. Barra: $1 \mathrm{~cm}$.

Figure 2. Seeds of Manilkara salzmannii (A. DC.) H. J. Lam: 2A and 2B - seed detail; 2C - embryo; 2D - cotyledon. Scale: $1 \mathrm{~cm}$.

Segundo Lorenzi (1992), a característica mais marcante na semente de Manilkara salzmannii é a presença de um conjunto de fibras na porção cotiledonar (periférica) das sementes, conferindo-lhe maior resistência e dureza, o que pode estar relacionado com a defesa a predadores e proteção após a ingestão dos frutos por animais dispersores, dos quais se destacam os morcegos. Barroso et al. (1999) 
consideraram que, em geral, as sementes das Sapotaceae são facilmente reconhecíveis, devido à testa brilhante e escura e ao hilo particular, como observado no presente estudo.

\section{CONCLUSÕES}

No intuito de subsidiar os estudos sobre Manilkara salzmannii, as características morfológicas tanto do fruto quanto da semente devem ser estudadas para as demais espécies do gênero, uma vez que esses dados podem servir como ferramenta para a sua identificação, como já são usados por diversos autores (BRAVATO, 1974; OLIVEIRA; BELTRATI, 1994; MELO et al., 2004) para outras espécies. Esse conhecimento apresenta também relevante valor ecológico, pois pode auxiliar na determinação da variabilidade da espécie (PINTO et al., 2003), bem como no estudo do tipo de dispersão e dos agentes dispersores. Além disso, essas informações podem ser utilizadas para auxiliar nas análises de sucessão e regeneração de ecossistemas florestais, uma vez que essa espécie pode ser indicada para reflorestamento de múltiplo uso.

\section{REFERÊNCIAS}

AMORIN, I. S.; DAVIDE, A. C.; CHAVES, M. M. Morfologia do fruto e da semente, e da germinação de Trema micrantha (L.) Blum. Cerne, Lavras, v. 3, n. 1, p. 129-142, 1997.

ANDRADE-LIMA, D. Estudos fitogeográficos de Pernambuco. Recife: IPA. 1957. (Nova Serie. Publicação 2).

ARAÚJO, R. R.; SANTOS, E. D.; LEMOS, E. E. P. Caracterização física e química de frutos de maçaranduba (Manilkara salzmannii, Sapotaceae). Trabalho apresentado no 20. CONGRESSO BRASILEIRO DE FRUTICULTURA; 54. ANNUAL MEETING OF THE INTERAMERICAN SOCIETY FOR TROPICAL HORTICULTURE. Vitória, 2008.

BARROSO, G. M.; MORIM, M. P.; PEIXOTO, A. L.; ICHASO, C. L. F. Frutos e sementes: morfologia aplicada à sistemática de dicotiledôneas. Viçosa: UFV, 1999. 443 p.

BRASIL, Ministério da Agricultura e Reforma Agrária. Regras para análise de sementes. Brasília, DF: SNDA/DNDV/CLAV, 1992. $365 \mathrm{p}$.

BRAVATO, M. Estudo morfológico de frutos e semillas de las Mimosoideae (Leguminosae) de Venezuela. Acta Botânica Venezuelica, Caracas, v. 9, n. 1-4, p. 317-361, 1974.

BUDOWSKI, G. Distribution of tropical American rain forest species in the light of sucessional processes. Turrialba, San Jose, CR, v. 15, n. 1, p. 40-42, 1965.

CRUZ, E.D.; MARTINS, F.O.; CARVALHO, J.E.U. Biometria de frutos e sementes e germinação de jatobá-curuba (Hymenaea intermedia Ducke, Leguminosae-Caesalpinioideae). Revista Brasileira de Botânica, São Paulo, v. 24, n. 2, p. 161-165, 2001.

KÖPPEN, W. Climatologia: con un estudio de los climas de la tierra. México: Fondo de Cultura Economica, 1948. 478 p.

LAWRENCE, G. H. M. Taxonomia das plantas vasculares. Lisboa: Fundação Cauloste Gulbenkiar., 1951. v. 2.

LORENZI, H. Árvores brasileiras: manual de identificação e cultivo de plantas arbóreas nativas do Brasil. Nova Odessa: Plantarum, 1992. v. 1.

MEDEIROS, D. P. W.; LOPES, A.; ZICKEL, C. S. Phenology of woody species in a tropical coastal vegetation, northeastern Brazil. Flora, London, v. 202, p. 235-247, 2007.

MELO, M. da G. G.; MENDONÇA, M. S. de; MENDES, A. M. da S. Análise morfológica de sementes, germinação e plântulas de jatobá (Hymenaea intermédia Ducke var. adenotricha (Ducke) Lee \& Lang.) (Leguminosae-caesalpinioideae). Acta Amazônica, Manuas, v. 34, n. 1, p. 9-14, 2004. 
OLIVEIRA, D. M. T. de; BELTRATI, C. M. Morfologia e anatomia dos frutos e sementes de Inga fagifolia Willd. (Fabaceae: Mimosoideae). Revista Brasileira de Biologia, Rio de Janeiro, v. 54, n. 1, p. 91-100, 1994.

PENNINGTON, T. D. Sapotaceae. In Flora Neotropica, New York. v. 52, 770 p., 1990.

PINTO, D. D.; MOURÃO, K. S. M.; SOUZA, L. A. de; MOSCHETA, I. S. Morfo-anatomia do fruto e da semente em desenvolvimento de Guarea macrophylla Vahl. (Meliaceae). Acta Cientifica Venezolana, Caracas, v. 54, p. 238-246, 2003.

RICARDI, S. M. H. Famílias de Dicotiledôneas Venezolonas I. 2. ed. Mérida: [s.n.], 1992. 170 p.

SOUZA, V. C.; LORENZI, H. Botânica sistemática: guia ilustrado para identificação das famílias de fanerógamas nativas e exóticas no Brasil, baseado em APG II. 2. ed. Nova Odessa: Plantarum. 2008. 704 p. 